\title{
Binary positive semidefinite matrices and associated integer polytopes
}

\author{
Adam N. Letchford • Michael M. Sørensen
}

Received: 10 September 2009 / Accepted: 9 April 2010 / Published online: 23 April 2010

C Springer and Mathematical Programming Society 2010

\begin{abstract}
We consider the positive semidefinite (psd) matrices with binary entries, along with the corresponding integer polytopes. We begin by establishing some basic properties of these matrices and polytopes. Then, we show that several families of integer polytopes in the literature - the cut, boolean quadric, multicut and clique partitioning polytopes - are faces of binary psd polytopes. Finally, we present some implications of these polyhedral relationships. In particular, we answer an open question in the literature on the max-cut problem, by showing that the rounded psd inequalities define a polytope.
\end{abstract}

Keywords Polyhedral combinatorics - Semidefinite programming · Max-cut problem · Clique partitioning problem · Quadratic 0-1 programming

Mathematics Subject Classification (2000) 90C22 - 90C27 · 90C57

\section{Introduction}

A real square symmetric matrix $M \in \mathbb{R}^{n \times n}$ is called positive semidefinite ( $\mathrm{psd}$ ) if and only if any of the following (equivalent) conditions hold:

- $b^{T} M b \geq 0$ for all $b \in \mathbb{R}^{n}$,

\footnotetext{
A. N. Letchford $(\varangle)$

Department of Management Science, Lancaster University, Lancaster LA1 4YW, UK e-mail: A.N.Letchford@lancaster.ac.uk

M. M. Sørensen

CORAL, Department of Business Studies, Aarhus School of Business, Aarhus University,

Fuglesangs Allé 4, 8210 Aarhus V, Denmark

e-mail:mim@asb.dk
} 


$$
\left(\begin{array}{llll}
1 & 0 & 0 & 1 \\
0 & 0 & 0 & 0 \\
0 & 0 & 1 & 0 \\
1 & 0 & 0 & 1
\end{array}\right)=\left(\begin{array}{cc}
0.6 & 0.8 \\
0 & 0 \\
0.8 & -0.6 \\
0.6 & 0.8
\end{array}\right)\left(\begin{array}{cccc}
0.6 & 0 & 0.8 & 0.6 \\
0.8 & 0 & -0.6 & 0.8
\end{array}\right)
$$

Fig. 1 A binary psd matrix and a factorisation

- all principal submatrices of $M$ have non-negative determinants,

- there exists a real matrix $A$ such that $M=A A^{T}$.

The set of psd matrices of order $n$ forms a convex cone in $\mathbb{R}^{n \times n}$ (e.g., [22]), and is often denoted by $\mathcal{S}_{+}^{n}$.

In this paper, we consider the binary psd matrices, i.e., psd matrices belonging to $\{0,1\}^{n \times n}$. Figure 1 shows an example of a binary psd matrix of order 4 , along with one of its factorisations. We also consider an associated family of integer polytopes, which we call binary psd polytopes.

Although psd matrices and semidefinite programming have received much interest from the integer programming and combinatorial optimisation community (see the surveys [17] and [24]), these specific matrices and polytopes appear to have received no attention. This is remarkable, because, as we will see, the matrices can be easily characterised, and they have a natural graphical interpretation. Moreover, several important and well-known integer polytopes-such as the cut, boolean quadric, multicut and clique partitioning polytopes - can in fact be viewed as nothing but faces of binary psd polytopes. In that sense, the binary psd polytopes form an important, and hitherto overlooked, family of 'master' polytopes for combinatorial optimisation.

The paper is structured as follows. Section 2 presents three characterisations of binary psd matrices, along with the graphical representation. Section 3 introduces the binary psd polytopes and presents some elementary results on their structure. In Sect. 4, we establish the relationships between the binary psd polytopes and the other four families of polytopes mentioned above. In Sect. 5, we present some implications of these polyhedral relationships. In particular, we answer an open question in the literature on the max-cut problem, by showing that the so-called rounded psd inequalities define a polytope. Finally, some concluding remarks are given in Sect. 6.

An extended abstract of this paper appeared in the 2008 IPCO proceedings [25].

\section{Binary Psd matrices}

This section is concerned with binary psd matrices. We characterise the matrices (Sect. 2.1), give a graphical representation (Sect. 2.2), and give an associated complexity result (Sect 2.3). 
Fig. 2 An alternative factorisation of the matrix shown in Fig. 1.

$$
\left(\begin{array}{llll}
1 & 0 & 0 & 1 \\
0 & 0 & 0 & 0 \\
0 & 0 & 1 & 0 \\
1 & 0 & 0 & 1
\end{array}\right)=\left(\begin{array}{ll}
1 & 0 \\
0 & 0 \\
0 & 1 \\
1 & 0
\end{array}\right)\left(\begin{array}{llll}
1 & 0 & 0 & 1 \\
0 & 0 & 1 & 0
\end{array}\right)
$$

\subsection{Characterisations}

We now give three characterisations of binary psd matrices. The first characterisation is in terms of symmetric rank one binary matrices. Note that the symmetric rank one binary matrices are precisely those matrices that can be written in the form $v v^{T}$ for some (non-zero) binary vector $v \in\{0,1\}^{n}$.

Proposition 1 A symmetric binary matrix is psd if and only if it is either the zero matrix or the sum of one or more symmetric rank one binary matrices.

Proof The 'if' part follows trivially from the fact that $\mathcal{S}_{+}^{n}$ is a cone. We prove the 'only if' part. Suppose that $M$ is a binary psd matrix that is not the zero matrix. Since all $2 \times 2$ principal submatrices of $M$ must have non-negative determinant, we have that, if $M_{i i}=0$ for some $i \in\{1, \ldots, n\}$, then $M_{i j}=M_{j i}=0$ for $j=1, \ldots, n$. Thus, if we let $R=\left\{i \in\{1, \ldots, n\}: M_{i i}=1\right\}$, we have that $M$ has zero entries outside the principal submatrix defined by the row/column indices in $R$. This submatrix, which must also be psd, has $1 \mathrm{~s}$ on the main diagonal. The fact that a binary psd matrix with $1 \mathrm{~s}$ on the main diagonal is the sum of symmetric rank one binary matrices is well-known and easy to prove: see, e.g., Lemma 1 of Dukanovic and Rendl [15].

The second characterisation follows easily:

Proposition 2 A symmetric binary matrix is psd if and only if it equals $A A^{T}$ for some binary matrix $A$.

Proof The 'if' part follows immediately from the definition of psd-ness. We show the 'only if' part. Let $M \in\{0,1\}^{n \times n}$ be a binary psd matrix. If $M$ is the zero matrix, the result is trivial. Otherwise, from Proposition 1, there exists a positive integer $r$ and vectors $v^{1}, \ldots, v^{r} \in\{0,1\}^{n}$ such that:

$$
M=\sum_{k=1}^{r} v^{k}\left(v^{k}\right)^{T} .
$$

If we let $A$ be the $n \times r$ matrix whose $k$ th column is the vector $v^{k}$, we have that $M=A A^{T}$.

For example, the binary psd matrix shown in Fig. 1 has the alternative factorisation shown in Fig. 2.

We note in passing the following corollary:

Corollary 1 Binary psd matrices are completely positive. That is, they are equal to $A A^{T}$ for some non-negative real matrix $A$. 
Completely positive matrices were introduced by Diananda [14] and Hall and Newman [21]. They have received increased attention recently, due to their connections with various $\mathcal{N} \mathcal{P}$-hard optimisation problems (see, e.g., [6] and [29]).

We now come to our third characterisation, which is in terms of linear inequalities:

Proposition 3 A symmetric binary matrix $M \in\{0,1\}^{n \times n}$, with $n \geq 3$, is psd if and only if it satisfies the following inequalities:

$$
\begin{aligned}
M_{i j} & \leq M_{i i} \quad(1 \leq i<j \leq n) \\
M_{i k}+M_{j k} & \leq M_{k k}+M_{i j} \quad(1 \leq i<j \leq n ; k \neq i, j) .
\end{aligned}
$$

Proof It is easy to check that the inequalities (1) and (2) are satisfied by symmetric rank one binary matrices. Proposition 1 then implies that they are satisfied by binary psd matrices. Now, suppose that a symmetric binary matrix $M$ satisfies the inequalities (1) and (2). If $M_{i i}=0$ for a given $i$, the inequalities (1) imply that $M_{i j}=M_{j i}=0$ for all $j \neq i$. Thus, just as in the proof of Proposition 1, we can assume that $M$ has $1 \mathrm{~s}$ on the main diagonal. Now note that, if $M_{i k}=M_{j k}=1$ for some indices $i, j, k$, then the inequalities (2) ensure that $M_{i j}=1$. By transitivity, this implies that $\{1, \ldots, n\}$ can be partitioned into subsets in such a way that, for all pairs $i, j, M_{i j}=1$ if and only if $i$ and $j$ belong to the same subset. That is to say, $M$ is the sum of one or more symmetric rank one binary matrices. By Proposition $1, M$ is psd.

\subsection{Graphical representation}

The binary psd matrices have a natural graphical representation, as we now explain. Let $K_{n}=\left(V_{n}, E_{n}\right)$ denote the complete graph of order $n$, where $V_{n}=\{1, \ldots, n\}$ is the vertex set and $E_{n}=\left\{S \subset V_{n}:|S|=2\right\}$ is the edge set. Given any $n \times n$ binary psd matrix $M$, we can construct a subgraph of $K_{n}$ as follows. The vertex $i$ is included in the subgraph if and only if $M_{i i}=1$, and the edge $\{i, j\}$ is included if and only if $M_{i j}=M_{j i}=1$.

If $M$ has rank one, the subgraph will consist of a vertex set $S$, where $S=\left\{i \in V_{n}\right.$ : $\left.M_{i i}=1\right\}$, together with all edges in $E_{n}$, if any, having both end-vertices in $S$. We call such a subgraph a clique subgraph. If $M$ has rank $r>1$, then the corresponding subgraph of $K_{n}$ will consist of the union of $r$ disjoint clique subgraphs.

Figure 3 illustrates this concept. The binary psd matrix on the left is of order 7 and has rank three. The corresponding subgraph of $K_{7}$ is shown on the right. The three clique subgraphs of which it is composed have vertex sets $\{1,2,4\},\{5,6\}$ and $\{7\}$. Note that vertex 3 is not included in a clique subgraph, since $M_{33}=0$.

\subsection{Complexity}

We now show that optimising a linear function over the set of binary psd matrices is $\mathcal{N P}$-hard. We do this by reduction from the so-called Clique Partitioning Problem. 


$$
\left(\begin{array}{lllllll}
1 & 1 & 0 & 1 & 0 & 0 & 0 \\
1 & 1 & 0 & 1 & 0 & 0 & 0 \\
0 & 0 & 0 & 0 & 0 & 0 & 0 \\
1 & 1 & 0 & 1 & 0 & 0 & 0 \\
0 & 0 & 0 & 0 & 1 & 1 & 0 \\
0 & 0 & 0 & 0 & 1 & 1 & 0 \\
0 & 0 & 0 & 0 & 0 & 0 & 1
\end{array}\right)
$$
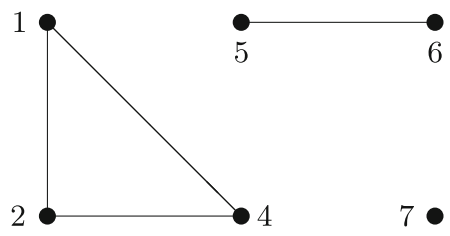

Fig. 3 A binary psd matrix and its graphical representation

Given any partition of $V_{n}$ into sets $S_{1}, \ldots, S_{r}$, the set of edges

$$
E_{n}\left(S_{1}, \ldots, S_{r}\right)=\left\{\{i, j\} \in E_{n}:\{i, j\} \subseteq S_{p} \text { for some } 1 \leq p \leq r\right\}
$$

is called a clique partition. Given a vector $w \in \mathbb{Q}^{\left|E_{n}\right|}$ of edge-weights, the Clique Partitioning Problem calls for a clique partition of maximum total edge-weight. The problem has applications in statistical clustering, and is $\mathcal{N} \mathcal{P}$-hard in the strong sense (Grötschel and Wakabayashi [19]).

Proposition 4 Optimising a linear function over the set of binary psd matrices is $\mathcal{N P}$-hard in the strong sense.

Proof It follows from the discussion in the previous subsection that a symmetric binary matrix $M$ of order $n$ is psd if and only if the edge set $\left\{\{i, j\} \in E_{n}: M_{i j}=1\right\}$ is a clique partition. Thus, solving the Clique Partitioning Problem for a given $n$ and $w$ is equivalent to maximising the linear function

$$
\sum_{1 \leq i<j \leq n} w_{i j} M_{i j}
$$

over the set of binary psd matrices of order $n$.

We remark that a similar hardness result holds for the symmetric rank one binary matrices. Indeed, a symmetric binary matrix has rank one if and only if it satisfies the quadratic equations $M_{i j}=M_{i i} M_{j j}$ for all pairs $i, j$. Thus, optimising a linear function over the symmetric rank one binary matrices is equivalent to unconstrained quadratic $0-1$ programming, which is also $\mathcal{N} \mathcal{P}$-hard in the strong sense [16].

Moreover, optimising a linear function over the set of psd matrices with \pm 1 entries is known to be equivalent to the max-cut problem, and therefore $\mathcal{N} \mathcal{P}$-hard in the strong sense $[18,23]$.

These connections between binary psd matrices and various $\mathcal{N} \mathcal{P}$-hard combinatorial optimisation problems have polyhedral implications, as will be shown in Sect. 4.

\section{Binary Psd polytopes}

This section is concerned with binary psd polytopes. We define them in Sect. 3.1, give some elementary results in Sect. 3.2, prove a general result about homogeneous 
Fig. 4 The polytope $\mathcal{P}_{2}$

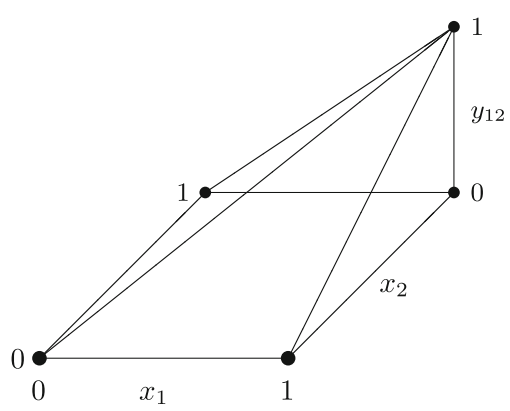

inequalities in Sect. 3.3, and introduce a large class of homogeneous inequalities in Sect. 3.4.

\subsection{Definitions}

Note that any binary psd matrix $M$, being symmetric, satisfies the $\left(\begin{array}{l}n \\ 2\end{array}\right)$ equations $M_{i j}=$ $M_{j i}$ for all $1 \leq i<j \leq n$. Therefore, if we defined the binary psd polytope in $\mathbb{R}^{n \times n}$, it would not be full-dimensional. Therefore, we decided to work in $\mathbb{R}^{V_{n} \cup E_{n}}$ instead.

Accordingly, we define for all $i \in V_{n}$ the binary variable $x_{i}$, which takes the value 1 if and only if $M_{i i}=1$; and we define, for all $\{i, j\} \in E_{n}$, the binary variable $y_{i j}$, which takes the value 1 if and only if $M_{i j}=M_{j i}=1$. We denote by $\mathcal{M}(x, y)$ the linear operator that maps a given pair $(x, y) \in\{0,1\}^{V_{n} \cup E_{n}}$ onto the corresponding $n \times n$ symmetric matrix. Then, the binary psd polytope of order $n$ is defined as:

$$
\mathcal{P}_{n}=\operatorname{conv}\left\{(x, y) \in\{0,1\}^{V_{n} \cup E_{n}}: \mathcal{M}(x, y) \in \mathcal{S}_{+}^{n}\right\} .
$$

Example For $n=2$, there are 5 binary psd matrices:

$$
\left(\begin{array}{ll}
0 & 0 \\
0 & 0
\end{array}\right)\left(\begin{array}{ll}
1 & 0 \\
0 & 0
\end{array}\right)\left(\begin{array}{ll}
0 & 0 \\
0 & 1
\end{array}\right)\left(\begin{array}{ll}
1 & 0 \\
0 & 1
\end{array}\right)\left(\begin{array}{ll}
1 & 1 \\
1 & 1
\end{array}\right)
$$

The corresponding vectors $\left(x_{1}, x_{2}, y_{12}\right)$ are $(0,0,0),(1,0,0),(0,1,0),(1,1,0)$ and $(1,1,1)$, respectively. The polytope $\mathcal{P}_{2}$ is displayed in Fig. 4 . It is described by the linear inequalities $x_{1} \leq 1, x_{2} \leq 1, y_{12} \geq 0, y_{12} \leq x_{1}$ and $y_{12} \leq x_{2}$.

Proposition 3 enables us to define $\mathcal{P}_{n}$ more explicitly.

Proposition 5 For $n \geq 3, \mathcal{P}_{n}$ is the convex hull of pairs $(x, y) \in\{0,1\}^{V_{n} \cup E_{n}}$ satisfying the following inequalities:

$$
\begin{aligned}
y_{i j} & \leq x_{i} \quad\left(i \in V_{n}, j \in V_{n} \backslash\{i\}\right) \\
y_{i k}+y_{j k} & \leq x_{k}+y_{i j} \quad\left(\{i, j\} \in E_{n}, k \in V_{n} \backslash\{i, j\}\right) .
\end{aligned}
$$

We will call the inequalities (3) and (4) variable upper bounds and triangle inequalities, respectively. 


\subsection{Some elementary results}

We now present some simple results about binary psd polytopes. First, we show that they are full-dimensional:

Lemma 1 For $n \geq 2, \mathcal{P}_{n}$ is full-dimensional, i.e., has dimension $\left(\begin{array}{c}n+1 \\ 2\end{array}\right)$.

Proof Consider the following extreme points of $\mathcal{P}_{n}$ :

- $\quad$ the origin (i.e., all variables set to zero);

- for each $i \in V_{n}$, the point having $x_{i}=1$, and all other variables set to zero;

- for each $\{i, j\} \in E_{n}$, the point having $x_{i}=x_{j}=y_{i j}=1$, and all other variables set to zero.

These $\left(\begin{array}{c}n+1 \\ 2\end{array}\right)+1$ points are easily shown to be affinely independent.

Next, we show that the upper bounds on the $x$ variables induce facets:

Lemma 2 For all $i \in V_{n}$, the upper bound $x_{i} \leq 1$ induces a facet of $\mathcal{P}_{n}$ :

Proof The upper bound $x_{i} \leq 1$ is satisfied at equality by the following points:

- the point having $x_{i}=1$, and all other variables set to zero;

- for each $j \in V_{n} \backslash\{i\}$, the point having $x_{i}=x_{j}=1$, and all other variables set to zero;

- for each $j \in V_{n} \backslash\{i\}$, the point having $x_{i}=x_{j}=y_{i j}=1$, and all other variables set to zero;

- for each $\{j, k\} \subset V_{n} \backslash\{i\}$, the point having $x_{i}=x_{j}=x_{k}=y_{j k}=1$, and all other variables zero.

These $\left(\begin{array}{c}n+1 \\ 2\end{array}\right)$ points are also easily shown to be affinely independent.

Finally, we show that facet-inducing inequalities have a special structure:

Proposition 6 Every inequality inducing a facet of $\mathcal{P}_{n}$, apart from the upper bounds $x_{i} \leq 1$ for all $i \in V_{n}$, can be written in the form $b^{T} y \leq a^{T} x+c$, with $a \geq 0$ and $c \geq 0$.

Proof Clearly, any facet-inducing inequality can be written in the form $b^{T} y \leq a^{T} x+c$. Now, the origin belongs to $\mathcal{P}_{n}$, which shows that $c \geq 0$. Moreover, suppose that the inequality is not an upper bound. Then, for each $i \in V_{n}$, there must exist a point $\left(x^{*}, y^{*}\right)$ lying on the facet, for which $x_{i}^{*}=0$. Now, the point obtained from $\left(x^{*}, y^{*}\right)$ by changing $x_{i}$ to 1 belongs to $\mathcal{P}_{n}$ (since changing $M_{i i}$ from zero to one preserves psd-ness). Therefore $a_{i} \geq 0$ for all $i \in V_{n}$. 


\subsection{Homogeneous valid inequalities}

An inequality $b^{T} y \leq a^{T} x+c$ is called homogeneous if $c=0$. In this subsection, we give a result concerned with homogeneous inequalities that define facets of $\mathcal{P}_{n}$. To do this, we need to recall the definition of the so-called boolean quadric cone.

Let us say that a vector $\left(x^{*}, y^{*}\right) \in\{0,1\}^{V_{n} \cup E_{n}}$ has rank one if the corresponding symmetric matrix $\mathcal{M}\left(x^{*}, y^{*}\right)$ has rank one. The boolean quadric cone (sometimes called the correlation cone) is the polyhedral cone in $\mathbb{R}^{V_{n} \cup E_{n}}$ consisting of all nonnegative linear combinations of rank one vectors (see [13]). We let $\mathrm{BQC}_{n}$ denote the boolean quadric cone of order $n$.

We have the following result:

Proposition 7 A homogeneous inequality is valid (or facet-inducing) for $\mathcal{P}_{n}$ if and only if it is valid (or facet-inducing) for $\mathrm{BQC}_{n}$.

Proof It follows from Proposition 1 that every rank one vector in $\{0,1\}^{V_{n} \cup E_{n}}$ is an extreme point of $\mathcal{P}_{n}$, and that every extreme point of $\mathcal{P}_{n}$ is a non-negative linear combination of rank one vectors in $\{0,1\} V_{n} \cup E_{n}$. Therefore, every valid inequality for $\mathrm{BQC}_{n}$ is valid for $\mathcal{P}_{n}$, and every homogeneous valid inequality for $\mathcal{P}_{n}$ is valid for $\mathrm{BQC}_{n}$. Now, since the origin is an extreme point of $\mathcal{P}_{n}$, a homogeneous valid inequality defines a facet of $\mathcal{P}_{n}$ if and only if it is not a convex combination of other homogeneous valid inequalities. This is the case if and only if it defines a facet of $\mathrm{BQC}_{n}$.

The boolean quadric cone has been studied in depth, and many valid and facetinducing inequalities are known (see again [13]). We will mention one large class of inequalities in the next subsection. For now, however, we simply note the following corollary of Proposition 7:

Corollary 2 The following homogeneous inequalities induce facets of $\mathcal{P}_{n}$ :

- $\quad$ The non-negativity inequalities $y_{e} \geq 0$ for all $e \in E_{n}$, when $n \geq 2$.

- $\quad$ The variable upper bounds (3), when $n \geq 2$.

- $\quad$ The triangle inequalities (4), when $n \geq 3$.

Proof All of these inequalities are known to induce facets of $\mathrm{BQC}_{n}$; see, e.g., Padberg [28].

We will mention some non-trivial inhomogeneous inequalities for $\mathcal{P}_{n}$ in Sect. 5.1.

\subsection{Hypermetric correlation inequalities}

Recall that a matrix $M$ is psd if and only if $b^{T} M b \geq 0$ for all $b \in \mathbb{R}^{n}$. This immediately implies that the following homogeneous inequalities are valid for $\mathcal{P}_{n}$ :

$$
\sum_{i \in V_{n}} b_{i}^{2} x_{i}+2 \sum_{\{i, j\} \in E_{n}} b_{i} b_{j} y_{i j} \geq 0 \quad\left(\forall b \in \mathbb{R}^{n}\right) .
$$


These inequalities are however dominated by the following stronger homogeneous inequalities, that are known to be valid for $\mathrm{BQC}_{n}$ (e.g., [4]):

$$
\sum_{i \in V_{n}} b_{i}\left(b_{i}-1\right) x_{i}+2 \sum_{\{i, j\} \in E_{n}} b_{i} b_{j} y_{i j} \geq 0 \quad\left(\forall b \in \mathbb{Z}^{n}\right) .
$$

We will follow Deza and Grishukhin [9] in calling the inequalities (5) hypermetric correlation inequalities.

To see that the hypermetric correlation inequalities are valid, note that $b^{T} x\left(b^{T} x-\right.$ $1) \geq 0$ for any integer vector $b$ and binary vector $x$, and therefore $b^{T} M b-$ $b^{T} \operatorname{diag}(M) \geq 0$ for any symmetric rank one binary matrix $M$.

Since the number of hypermetric correlation inequalities is infinite, not all of them induce facets of $\mathrm{BQC}_{n}$. A survey of necessary and sufficient conditions for them to induce facets can be found in Deza and Laurent [13]. We remark that Padberg [28] characterised the facet-inducing hypermetric correlation inequalities with $b \in\{0, \pm 1\}^{n}$. All of the inequalities mentioned in Corollary 2 are of this type.

\section{Relationships with other polytopes}

In this section, we explain in detail how the binary psd polytopes are related to certain other well-known polytopes in combinatorial optimisation. We will use these polyhedral relationships to prove several new results in Sect. 5.

\subsection{The clique partitioning polytope}

The clique partitioning polytope [19] is the polytope associated with the Clique Partitioning Problem, mentioned in Sect. 2.3. It is defined as:

$$
\operatorname{conv}\left\{y \in\{0,1\}^{E_{n}}: y_{i k}+y_{j k} \leq y_{i j}+1 \quad\left(\forall\{i, j\} \in E_{n}, k \in V_{n} \backslash\{i, j\}\right)\right\} .
$$

We will let $\mathrm{CPP}_{n}$ denote the clique partitioning polytope of order $n$. From the discussion in Sect. 2, a vector $y \in\{0,1\}^{E_{n}}$ is a vertex of $\mathrm{CPP}_{n}$ if and only if there exists a vector $x \in\{0,1\}^{V_{n}}$ such that $(x, y) \in \mathcal{P}_{n}$. Thus:

Proposition $8 \mathrm{CPP}_{n}$ is the projection of $\mathcal{P}_{n}$ onto $\mathbb{R}^{E_{n}}$.

As an immediate corollary we have:

Corollary 3 If the inequality $b^{T} y \leq a^{T} x+c$ is valid for $\mathcal{P}_{n}$, then the inequality $b^{T} y \leq \sum_{i \in V_{n}} a_{i}+c$ is valid for $\mathrm{CPP}_{n}$.

In fact, we can say something stronger.

Proposition $9 \mathrm{CPP}_{n}$ is congruent to a face of $\mathcal{P}_{n}$.

Proof From Lemma 2, the following $n$ linear inequalities induce facets of $\mathcal{P}_{n}$ :

$$
x_{i} \leq 1 \quad\left(i \in V_{n}\right) \text {. }
$$


Let $F$ be the face of $\mathcal{P}_{n}$ obtained by setting these inequalities at equality, and let $\left(x^{*}, y^{*}\right) \in\{0,1\}^{V_{n} \cup E_{n}}$ be an extreme point of $F$. From the discussion in Sect. 2.2, the edges with $y_{i j}^{*}=1$ form a clique partition. Therefore, $y^{*}$ is an extreme point of $\mathrm{CPP}_{n}$. Moreover, if $y^{*}$ is any extreme point of $\mathrm{CPP}_{n}$, we obtain an extreme point $\left(x^{*}, y^{*}\right)$ of $F$ simply by setting $x_{i}^{*}=1$ for all $i \in V_{n}$. Thus, $\mathrm{CPP}_{n}$ is congruent to $F$.

An immediate consequence of Proposition 9 is that inequalities for $\mathrm{CPP}_{n}$ can be lifted to yield inequalities for $\mathcal{P}_{n}$ :

Proposition 10 Let $b^{T} y \leq c$ be a facet-inducing inequality for the clique partitioning polytope $\mathrm{CPP}_{n}$. Then there exists at least one facet-inducing inequality for $\mathcal{P}_{n}$ of the form

$$
b^{T} y \leq \sum_{i \in V_{n}} \alpha_{i} x_{i}+c-\sum_{i \in V_{n}} \alpha_{i}
$$

where $\alpha_{i} \geq 0$ for all $i \in V_{n}$.

\subsection{The boolean quadric polytope}

The boolean quadric polytope [28] of order $n$ is defined as:

$$
\mathrm{BQP}_{n}=\operatorname{conv}\left\{(x, y) \in\{0,1\}^{V_{n} \cup E_{n}}: y_{i j}=x_{i} x_{j}\left(\{i, j\} \in E_{n}\right)\right\} .
$$

The boolean quadric polytope, sometimes called the correlation polytope, arises naturally in quadratic $0-1$ programming, and also has many applications in statistics, probability and theoretical physics (see [13]).

Note that a pair $\left(x^{*}, y^{*}\right)$ is an extreme point of $\mathrm{BQP}_{n}$ if and only if it has rank one, i.e., if and only if $\mathcal{M}\left(x^{*}, y^{*}\right)$ is a symmetric rank one binary matrix. Therefore, $\mathrm{BQP}_{n} \subset \mathcal{P}_{n} \subset \mathrm{BQC}_{n}$. Together with Proposition 7, this implies the following:

Proposition $11 \mathrm{BQP}_{n}$ and $\mathcal{P}_{n}$ have the same homogeneous facets; i.e., an inequality $b^{T} y \leq a^{T} x$ is facet-inducing for $\mathrm{BQP}_{n}$ if and only if it is facet-inducing for $\mathcal{P}_{n}$.

In fact, the relationship between $\mathrm{BQP}_{n}$ and $\mathcal{P}_{n}$ goes deeper than this:

Proposition $12 \mathrm{BQP}_{n}$ is congruent to a face of $\mathcal{P}_{n+1}$.

Proof From Lemma 2 and Corollary 2, the following $n+1$ linear inequalities induce facets of $\mathcal{P}_{n+1}$ :

$$
\begin{aligned}
x_{n+1} & \leq 1 \\
y_{i, n+1} & \leq x_{i} \quad\left(i \in V_{n}\right) .
\end{aligned}
$$

Let $F$ be the face of $\mathcal{P}_{n+1}$ obtained by setting these inequalities at equality, and let $\left(x^{*}, y^{*}\right) \in\{0,1\}^{V_{n+1} \cup E_{n+1}}$ be an extreme point of $F$. From the triangle inequalities (4) with $k=n+1$ we have:

$$
y_{i, n+1}^{*}+y_{j, n+1}^{*} \leq x_{n+1}^{*}+y_{i j}^{*} \quad\left(\{i, j\} \in E_{n}\right) .
$$


Since by assumption $x_{n+1}^{*}=1$ and $y_{i, n+1}^{*}=x_{i}^{*}$ for all $i$, we have $x_{i}^{*}+x_{j}^{*} \leq 1+y_{i j}^{*}$ for all $\{i, j\} \in E_{n}$. Together with the variable upper bounds (3) and the fact that $x^{*}$ is binary, this implies that $y_{i j}^{*}=x_{i}^{*} x_{j}^{*}$ for all $\{i, j\} \in E_{n}$. Thus, if we project $\left(x^{*}, y^{*}\right)$ onto $\mathbb{R}^{V_{n} \cup E_{n}}$, we obtain an extreme point of $\mathrm{BQP}_{n}$. Using a similar argument, given any extreme point of $\mathrm{BQP}_{n}$, one can construct a unique extreme point of $F$ of which it is the projection. Thus, $\mathrm{BQP}_{n}$ is congruent to $F$.

The proof of Proposition 12 was inspired by the construction used by Lovász and Schrijver [26] for forming SDP relaxations of 0-1 Linear Programs.

Proposition 12 implies that valid inequalities for $\mathcal{P}_{n+1}$ can be converted into valid inequalities for $\mathrm{BQP}_{n}$, and that facet-inducing inequalities for $\mathrm{BQP}_{n}$ can be lifted to yield facet-inducing inequalities for $\mathcal{P}_{n+1}$. For the sake of brevity, we do not state these results formally.

\subsection{The cut and multicut polytopes}

Finally, we mention connections between the above polytopes and the cut and multicut polytopes.

Given any $S \subseteq V_{n}$, the set of edges

$$
\delta_{n}(S)=\left\{\{i, j\} \in E_{n}: i \in S, j \in V_{n} \backslash S\right\}
$$

is called an edge cutset or simply cut. The cut polytope $\mathrm{CUT}_{n}$ is the convex hull of the incidence vectors of all cuts in $K_{n}$ [3], i.e.,

$$
\mathrm{CUT}_{n}=\operatorname{conv}\left\{y \in\{0,1\}^{E_{n}}: \exists S \subset V_{n}: y_{e}=1 \Longleftrightarrow e \in \delta_{n}(S)\left(\forall e \in E_{n}\right)\right\}
$$

Similarly, given any partition of $V_{n}$ into sets $S_{1}, \ldots, S_{r}$, the set of edges

$$
\delta_{n}\left(S_{1}, \ldots, S_{r}\right)=\left\{\{i, j\} \in E_{n}: i \in S_{p}, j \in S_{q} \text { for some } p \neq q\right\}
$$

is called a multicut. The multicut polytope $\mathrm{MCUT}_{n}$ is defined accordingly (e.g., [11]).

We now recall two important facts about the cut polytope. First, the cut polytope $\mathrm{CUT}_{n+1}$ is the image of the boolean quadric polytope $\mathrm{BQP}_{n}$ under an affine mapping known as the covariance mapping (see [13]). This means that there is a one-to-one correspondence between the facets of the respective polytopes. This correspondence is the following [7]:

Proposition 13 Let $a \in \mathbb{R}^{V_{n}}, b \in \mathbb{R}^{E_{n}}, c \in \mathbb{R}^{E_{n+1}}$ be linked by:

$$
\left\{\begin{array}{clrl}
c_{i, n+1}=a_{i}+ & \frac{1}{2} \sum_{j \in V_{n} \backslash\{i\}} b_{i j} & & \text { for } i \in V_{n} \\
c_{e} & =-\frac{1}{2} b_{e} & & \text { for } e \in E_{n}
\end{array}\right.
$$

Given $a_{0} \in \mathbb{R}$, the inequality $c^{T} y \leq a_{0}$ is valid (resp. facet-inducing) for $\mathrm{CUT}_{n+1}$ if and only if the inequality $a^{T} x+b^{T} y \leq a_{0}$ is valid (resp. facet-inducing) for $\mathrm{BQP}_{n}$. 
Second, the cut polytope possesses a remarkable symmetry, via the so-called switching operation $[3,12,28]$. Specifically, if an inequality of the form

$$
\sum_{e \in E_{n}} \alpha_{e} y_{e} \leq \beta
$$

induces a facet of $\mathrm{CUT}_{n}$, then so does the 'switched' inequality

$$
\sum_{e \in E_{n} \backslash \delta(S)} \alpha_{e} y_{e}-\sum_{e \in \delta(S)} \alpha_{e} y_{e} \leq \beta-\sum_{e \in \delta(S)} \alpha_{e}
$$

for all $S \subset V_{n}$. Moreover, if one is given a list of all homogeneous inequalities that induce facets of $\mathrm{CUT}_{n}$, then by applying switching, one can obtain a complete linear description of $\mathrm{CUT}_{n}$.

We remark that the cut polytope is also equivalent (under a simple linear mapping) to the convex hull of the psd matrices with \pm 1 entries $[18,23]$.

As for the multicut polytope $\mathrm{MCUT}_{n}$, it is not hard to see that it is nothing but the complement of the clique partitioning polytope $\mathrm{CPP}_{n}$. That is, the vector $y^{*}$ belongs to $\mathrm{MCUT}_{n}$ if and only if the vector $\tilde{y}$, with $\tilde{y}_{e}=1-y_{e}^{*}$ for all $e \in E_{n}$, belongs to $\mathrm{CPP}_{n}$. This enables one to easily map valid inequalities and facets of $\mathrm{CPP}_{n}$ onto valid inequalities and facets of $\mathrm{MCUT}_{n}$, and vice-versa.

Finally, we 'complete the circle' of results by mentioning a link between the cut and multicut polytopes:

Proposition $14 \mathrm{CUT}_{n}$ and $\mathrm{MCUT}_{n}$ have the same homogeneous facets.

This fact was pointed out by Deza et al. [11].

\subsection{Summary of the polyhedral relationships}

In Fig. 5, we summarize the relationships between the five polytopes as established by Propositions 8-14. (Note that Proposition 11 is not displayed.) As we remarked in the introduction, the binary psd polytope $\mathcal{P}_{n}$ is the most complex of the five polytopes under discussion, in the sense that a complete description of $\mathcal{P}_{n}$ can be used to derive a complete description of the other four polytopes. We point out, however, that the multicut and clique partitioning polytopes are themselves more complex than the cut and boolean quadric polytopes: one needs only the homogeneous facets of $\mathrm{MCUT}_{n}$, together with the switching symmetry mentioned in the previous subsection, to obtain a complete description of $\mathrm{CUT}_{n}$ (and therefore, by the covariance mapping, also of $\left.\mathrm{BQP}_{n-1}\right)$.

\section{Consequences of the polyhedral relationships}

In this section, we consider some implications of the polyhedral relationships established in the previous section. In Sect. 5.1, we show how inhomogeneous inequalities 
Fig. 5 A pentagon of polyhedral relations

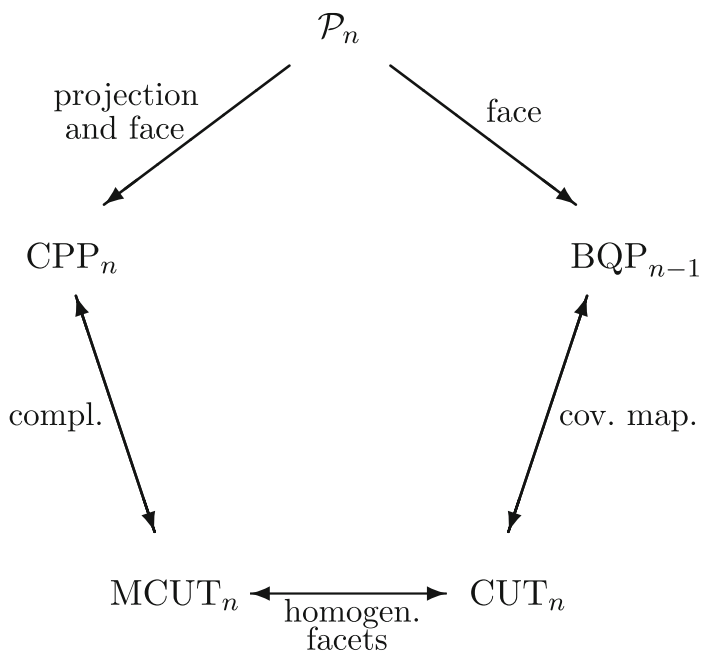

for binary psd polytopes can be derived from inequalities for clique partitioning polytopes. In Sect. 5.2, we show how new valid inequalities for the other polytopes can also be derived using similar arguments. Finally, in Sect. 5.3, we examine the hypermetric correlation inequalities, and some related inequalities, which will enable us to answer an open question in the literature on the max-cut problem.

\subsection{Inhomogeneous inequalities for binary psd polytopes}

In Sect. 3.3, we pointed out that all homogeneous valid inequalities for $\mathcal{P}_{n}$ come from the boolean quadric cone. We now show that, using the results in Sect. 4.1, one can derive interesting inhomogeneous inequalities for $\mathcal{P}_{n}$ from valid inequalities for $\mathrm{CPP}_{n}$.

We start with the so-called 2-chorded odd cycle inequalities of Grötschel and Wakabayashi [19]. These inequalities, which always induce facets of $\mathrm{CPP}_{n}$, take the form

$$
\sum_{e \in C} y_{e}-\sum_{e \in \bar{C}} y_{e} \leq(|C|-1) / 2
$$

where $C \subset E_{n}$ is the edge set of a simple cycle of odd length at least 5, and $\bar{C}$ is the set of 2-chords of $C$. It turns out that these inequalities induce facets of $\mathcal{P}_{n}$ :

Proposition 15 All 2-chorded odd cycle inequalities induce facets of $\mathcal{P}_{n}$.

Proof It suffices to show that the correct value for all of the lifting coefficients $\alpha_{i}$ in Proposition 10 is zero. This is trivial for the nodes not incident on an edge in $C$. So let $u$ be a node incident on an edge in $C$. We can obtain an extreme point of $\mathcal{P}_{n}$ satisfying the 2-chorded cycle inequality at equality by setting $x_{i}$ to one for all nodes apart from $u$, and setting $y_{e}$ to zero for all edges apart from $(|C|-1) / 2$ node-disjoint edges in the cycle $C$. Since we can obtain another extreme point of $\mathcal{P}_{n}$ by changing the value of $x_{u}$ from zero to one, $\alpha_{u}$ must be zero. 
Note that the 2-chorded odd cycle inequalities do not involve the $x$ variables. By lifting different inequalities, however, one can obtain inhomogeneous inequalities that involve the $x$ variables. We know of several examples, but give just one, for the sake of brevity. One can check (either by enumeration or with the aid of a computer) that the following inequality defines a facet of $\mathrm{CPP}_{7}$ :

$$
\begin{aligned}
& y_{12}+y_{14}+y_{17}+y_{23}+y_{34}+y_{47}+2 y_{57}+2 y_{67} \\
& -y_{13}-2 y_{16}-y_{24}-y_{27}-y_{37}-2 y_{45}-2 y_{56} \leq 4 .
\end{aligned}
$$

One can also check that the lifting coefficient $\alpha_{i}$ is equal to 2 for node 7 , and to 0 for all other nodes. Thus, the lifted inequality has a right-hand side of 2 and involves $x_{7}$.

We remark that not all inhomogeneous facets of $\mathcal{P}_{n}$ can be obtained by lifting facets of $\mathrm{CPP}_{n}$. For example, one can check that the following inhomogeneous inequality induces a facet of $\mathcal{P}_{4}$, yet cannot be obtained by lifting a facet of $\mathrm{CPP}_{4}$ :

$$
2 y_{13}+2 y_{14}+y_{23}+y_{24} \leq 1+2 x_{1}+2 y_{12}+y_{34} \text {. }
$$

5.2 New inequalities for the other polytopes

Using the chain of polyhedral relationships presented in the previous section, one can derive new valid inequalities not only for $\mathcal{P}_{n}$, but also for all of the other polytopes mentioned.

Here is an example. Grötschel and Wakabayashi [19] introduced a class of valid inequalities for $\mathrm{CPP}_{n}$ called 2-chorded path inequalities, which were generalised by Sørensen [30] as follows. Let $\left\{v_{1}, \ldots, v_{k}\right\}$ be a subset of $V_{n}$ with $k \geq 5$, and let $Z$ be a non-empty subset of $V_{n} \backslash\left\{v_{1}, \ldots, v_{k}\right\}$. Then the inequality

$$
\begin{aligned}
& \sum_{i=1}^{k-1} y_{v_{i}, v_{i+1}}-\sum_{i=1}^{k-2} y_{v_{i}, v_{i+2}}+\sum_{i=1}^{\lfloor k / 2\rfloor} \sum_{p \in Z} y_{p, v_{2 i}} \\
& \quad-\sum_{i=1}^{\lceil k / 2\rceil} \sum_{p \in Z} y_{p, v_{2 i-1}}-\left\lfloor\frac{k+2}{4}\right\rfloor \sum_{\{i, j\} \subset Z} y_{i j} \leq\lfloor k / 2\rfloor
\end{aligned}
$$

is valid for $\mathrm{CPP}_{n}$, and induces a facet of $\mathrm{CPP}_{n}$ unless $k$ is even and $|Z|<2$. Applying Proposition 10, we obtain (after some work) the following result:

Proposition 16 Suppose we take the 2-chorded path inequality (6) and replace the right-hand side with $\sum_{i=1}^{\lfloor k / 2\rfloor} x_{v_{2 i}}$. The resulting lifted 2 -chorded path inequality induces a facet of $\mathcal{P}_{n}$ if $k \geq 5, Z \neq \emptyset$ and, if $k$ is even, $|Z| \geq 2$.

Now, note that the lifted 2-chorded path inequalities are homogeneous. Therefore, by Propositions 7 and 11, they also induce facets of the boolean quadric cone $\mathrm{BQC}_{n}$ and boolean quadric polytope $\mathrm{BQP}_{n}$. Moreover, by applying the covariance mapping (Proposition 13), one can obtain facet-inducing inequalities for the cut polytope $\mathrm{CUT}_{n+1}$. These inequalities are also homogeneous, and therefore (by Proposition 14), 
they induce facets of the multicut polytope $\mathrm{MCUT}_{n+1}$ as well. Finally, by complementing, one obtains facet-inducing inequalities for $\mathrm{CPP}_{n+1}$. As far as we know, this gives new results for all polytopes mentioned.

As another example we consider the general 2-partition inequalities obtained by Grötschel and Wakabayashi [20] for $\mathrm{CPP}_{n}$. Actually, they describe two classes of general 2-partition inequalities. For the sake of brevity, we consider the ones described in Grötschel and Wakabayashi [20, Theorem 3.5], as the other ones can be treated in a similar manner. These inequalities take the form

$$
\begin{aligned}
& \sum_{k=1}^{p}\left(\sum_{i \in S_{k}} \sum_{j \in T_{k} \cup T^{\prime}} y_{i j}-\sum_{i \in S_{k}} \sum_{j \in S_{\ell}, \ell>k} y_{i j}-\sum_{\{i, j\} \subset S_{k}} y_{i j}\right) \\
& \quad-\sum_{\{i, j\} \subset T^{\prime}} y_{i j}-\sum_{k=1}^{p}\left(\sum_{\{i, j\} \subset T_{k}} y_{i j}+\sum_{i \in T_{k}} \sum_{j \in T^{\prime}} y_{i j}\right) \leq \sum_{k=1}^{p}\left|S_{k}\right|,
\end{aligned}
$$

where $S_{1}, \ldots, S_{p}, T_{1}, \ldots, T_{p}, T^{\prime}$, with $p \geq 2$, are mutually disjoint subsets of $V_{n}$ such that $1 \leq\left|S_{k}\right| \leq\left|T_{k}\right|$, for $k=1, \ldots, p$, and $\left|T^{\prime}\right| \geq 2$. Applying Proposition 10 we obtain (again after some work) the following result:

Proposition 17 Suppose we take the general 2-partition inequality (7) and replace the right-hand side with $\sum_{k=1}^{p} \sum_{i \in S_{k}} x_{i}$. The resulting lifted general 2-partition inequality induces a facet of $\mathcal{P}_{n}$.

As in the case of the lifted 2-chorded path inequalities, we see that the lifted general 2-partition inequalities are homogeneous. Thus, using the chain of polyhedral relationships, one can convert the latter into new facet-inducing inequalities for $\mathrm{BQC}_{n}, \mathrm{BQP}_{n}, \mathrm{CUT}_{n+1}, \mathrm{MCUT}_{n+1}$ and $\mathrm{CPP}_{n+1}$.

By applying a similar argument to other known valid inequalities for $\mathrm{CPP}_{n}$ and $\operatorname{MCUT}_{n}$, taken from [11,19,20,27,30,31], one can obtain further new inequalities for the various polytopes.

\subsection{On the hypermetric correlation inequalities}

Now we revisit the hypermetric correlation inequalities (5). We begin by noting that the hypermetric correlation inequalities for $\mathcal{P}_{n+1}$ imply, via Proposition 12 , the validity of the following inequalities for $\mathrm{BQP}_{n}$ :

$$
\sum_{i \in V_{n}} b_{i}\left(2 b_{n+1}+b_{i}-1\right) x_{i}+2 \sum_{\{i, j\} \in E_{n}} b_{i} b_{j} y_{i j} \geq b_{n+1}\left(1-b_{n+1}\right) \quad\left(\forall b \in \mathbb{Z}^{n+1}\right) .
$$

To our knowledge, these inequalities were first discovered by Boros and Hammer [5]. Note that the hypermetric correlation inequalities themselves are a special case of the inequalities (8), obtained when $b_{n+1}=0$. 
Next, we note that the hypermetric correlation inequalities for $\mathcal{P}_{n-1}$ correspond, under the covariance mapping, to the following valid inequalities for $\mathrm{CUT}_{n}$ and $\mathrm{MCUT}_{n}$ :

$$
\sum_{\{i, j\} \in E_{n}} a_{i} a_{j} y_{i j} \leq 0 \quad\left(\forall a \in \mathbb{Z}^{n}: \sum_{i=1}^{n} a_{i}=1\right)
$$

These are the well-known hypermetric inequalities, which have been studied in depth by Deza and colleagues (e.g., [8,10,12,13]).

If one applies the covariance mapping to the more general inequalities (8) instead, one obtains the following inequalities for $\mathrm{CUT}_{n}$ :

$$
\sum_{\{i, j\} \in E_{n}} a_{i} a_{j} y_{i j} \leq\left\lfloor\sigma(a)^{2} / 4\right\rfloor \quad\left(\forall a \in \mathbb{Z}^{n}: \sigma(a) \text { odd }\right)
$$

where $\sigma(a)=\sum_{i \in V_{n}} a_{i}$. These inequalities, which include the hypermetric inequalities as a special case, are also well-known in the literature on the cut polytope $[2,13]$. We will call them rounded psd inequalities.

We now present three new results. The first result, inspired by Proposition 12, is that the separation problem for the Boros-Hammer inequalities (8) can be reduced to the separation problem for the hypermetric correlation inequalities (5):

Proposition 18 Given a vector $\left(x^{*}, y^{*}\right) \in[0,1]^{V_{n} \cup E_{n}}$, let $(\tilde{x}, \tilde{y}) \in[0,1]^{V_{n+1} \cup E_{n+1}}$ be defined as follows. Let $\tilde{x}_{i}=x_{i}^{*}$ for $i \in V_{n}$, but let $\tilde{x}_{n+1}=1$. Let $\tilde{y}_{e}=y_{e}^{*}$ for e $\in E_{n}$, but let $\tilde{y}_{i, n+1}=x_{i}^{*}$ for $i \in V_{n}$. Then $\left(x^{*}, y^{*}\right)$ satisfies all Boros-Hammer inequalities (8) if and only if $(\tilde{x}, \tilde{y})$ satisfies all hypermetric correlation inequalities (5).

Proof Suppose that $(\tilde{x}, \tilde{y})$ violates a hypermetric correlation inequality. Then there exists a vector $b \in \mathbb{Z}^{n+1}$ such that

$$
\sum_{i \in V_{n+1}} b_{i}\left(b_{i}-1\right) \tilde{x}_{i}+2 \sum_{\{i, j\} \in E_{n+1}} b_{i} b_{j} \tilde{y}_{i j}<0 .
$$

From the construction of $(\tilde{x}, \tilde{y})$ this implies that:

$$
\sum_{i \in V_{n}} b_{i}\left(2 b_{n+1}+b_{i}-1\right) x_{i}^{*}+2 \sum_{\{i, j\} \in E_{n}} b_{i} b_{j} y_{i j}^{*}<b_{i}\left(1-b_{i}\right) \text {. }
$$

That is, $\left(x^{*}, y^{*}\right)$ violates a Boros-Hammer inequality. The reverse direction is similar.

Our second result is that the separation problem for the rounded psd inequalities (10) can be reduced to the separation problem for the hypermetric inequalities (9). (In fact, this follows from Proposition 18 and the covariance mapping. Nevertheless, we give an independent constructive proof, for the sake of clarity). 
Proposition 19 Given a vector $y^{*} \in[0,1]^{E_{n}}$, let $\tilde{y} \in[0,1]^{E_{n+1}}$ be defined as follows. Let $\tilde{y}_{e}=y_{e}^{*}$ for $e \in E_{n}$, but let $\tilde{y}_{i, n+1}=1-y_{i, n}^{*}$ for $i \in V_{n-1}$, and let $\tilde{y}_{n, n+1}=1$. Then $y^{*}$ satisfies all rounded psd inequalities (10) if and only if $\tilde{y}$ satisfies all hypermetric inequalities (9).

Proof Suppose that $\tilde{y}$ violates a hypermetric inequality. Then there exists a vector $\tilde{a} \in \mathbb{Z}^{n+1}$ such that $\sum_{i=1}^{n+1} \tilde{a}_{i}=1$ and such that $\sum_{\{i, j\} \in E_{n+1}} \tilde{a}_{i} \tilde{a}_{j} \tilde{y}_{i j}>0$. Now define a vector $a^{*} \in \mathbb{Z}^{n}$ as follows: $a_{i}^{*}=\tilde{a}_{i}$ for $i=1, \ldots, n-1$, and $a_{n}^{*}=\tilde{a}_{n}-\tilde{a}_{n+1}$. One can check that $\sum_{i=1}^{n+1} a_{i}^{*}$ is odd and that $\sum_{\{i, j\} \in E_{n}} a_{i}^{*} a_{j}^{*} y_{i j}^{*}>0$, and therefore $y^{*}$ violates a rounded psd inequality. The reverse direction is similar.

In one sense, Propositions 18 and 19 do not help much, since the complexity of hypermetric separation is a long-standing open problem (see, e.g., Deza and Laurent [13] and Avis [1]). They do however imply that a separation heuristic for any of the four classes of inequalities can be easily converted into separation heuristics for the other three classes. Moreover, Proposition 19 can be used to prove the following result:

Corollary 4 The rounded psd inequalities (10) define a polytope. (That is, although the inequalities are infinite in number, there exists a finite subset of them that dominates all the others).

Proof The hypermetric inequalities were shown to define a polyhedral cone by Deza et al. [10]. Now, Proposition 19 shows that the convex set defined by the rounded psd inequalities can be obtained by intersecting the hypermetric cone in $\mathbb{R}^{E_{n+1}}$ with the affine space defined by the equations $y_{i, n}+y_{i, n+1}=1$ for $i \in V_{n-1}$ and the equation $y_{n, n+1}=1$, and projecting the resulting polyhedron onto $\mathbb{R}^{E_{n}}$. The convex set is therefore a polyhedron. Moreover, it is bounded, since the inequalities $0 \leq y_{e} \leq 1$ for all $e \in E_{n}$ are themselves rounded psd inequalities.

This answers in the affirmative a question raised by Avis and Umemoto [2].

To close this section, we note that the hypermetric correlation inequalities (5) imply, via Corollary 3 , the following inequalities for $\mathrm{CPP}_{n}$ :

$$
\sum_{\{i, j\} \in E_{n}} b_{i} b_{j} y_{i j} \geq \frac{1}{2} \sum_{i \in V_{n}} b_{i}\left(1-b_{i}\right) \quad\left(\forall b \in \mathbb{Z}^{n}\right) .
$$

These inequalities generalise the weighted $(s, T)$-inequalities of Oosten et al. [27]. Moreover, if we complement them, we obtain the following inequalities for $\mathrm{MCUT}_{n}$ :

$$
\sum_{\{i, j\} \in E_{n}} b_{i} b_{j} y_{i j} \leq \sigma(b)(\sigma(b)-1) / 2 \quad\left(\forall b \in \mathbb{Z}^{n}\right) .
$$

The validity of these inequalities for $\mathrm{MCUT}_{n}$ was observed by Deza and Laurent [13] (p. 465). Note that they generalise the hypermetric inequalities (9), but they are generally weaker than the rounded psd inequalities (which are not valid in general for $\left.\operatorname{MCUT}_{n}\right)$. 


\section{Conclusions}

We have shown that the binary psd matrices are easily characterised and have a natural graphical representation. We have also shown that binary psd polytopes form an interesting family of 'master polytopes', that enable one to easily derive both known and new results for several other families of integer polytopes. We therefore believe that the binary psd polytopes deserve further attention.

One interesting topic for future research would be to study the complexity of the separation problem for various valid inequalities for binary psd polytopes. Positive separation results in this area would of course imply positive separation results for the other polytopes as well. A major open question is the complexity of separation for the hypermetric correlation inequalities and their variants.

Acknowledgments The research of the first author was supported by the Engineering and Physical Sciences Research Council under grant EP/D072662/1.

\section{References}

1. Avis, D.: $\mathrm{n}$ the complexity of testing hypermetric, negative type, $k$-gonal and gap inequalities. In: Akiyama, J., Kano, M. (eds.) Discrete and Computational Geometry. Lecture Notes in Computer Science vol. 2866, Springer, Berlin (2003)

2. Avis, D., Umemoto, J.: Stronger linear programming relaxations for max-cu. Math. Program. 97, 451469 (2003)

3. Barahona, F., Mahjoub, A.R.: On the cut polytope. Math. Program. 36, 151-173 (1986)

4. Boros, E., Hammer, P.L.: The max-cut problem and quadratic 0-1 optimization; polyhedral aspects, relaxations and bounds. Ann. Oper. Res. 33, 151-225 (1991)

5. Boros, E., Hammer, P.L.: Cut-polytopes, Boolean quadric polytopes and nonnegative quadratic pseudoBoolean functions. Math. Oper. Res. 18, 245-253 (1993)

6. Burer, S.: On the copositive representation of binary and continuous nonconvex quadratic programs. Math. Program. 120, 479-495 (2009)

7. De Simone, C.: The cut polytope and the Boolean quadric polytope. Discr. Math. 79, 71-75 (1989)

8. Deza, M.: Matrices de formes quadratique non négative pour des arguments binaires. C. R. Acad. Sci. Paris 277, 873-875 (1973)

9. Deza, M., Grishukhin, V.P. : Voronoi L-decomposition of $P S D_{n}$ and the hypermetric correlation cone. In: Engel, P., Syta, H. (eds.) Voronoi's Impact on Modern Science, Kiev: Institute of Mathematics of the National Academy of Science, Ukraine (1998)

10. Deza, M., Grishukhin, V.P., Laurent, M.: The hypermetric cone is polyhedral. Combinatorica 13, 397411 (1993)

11. Deza, M., Grötschel, M., Laurent, M.: Clique-web facets for multicut polytopes. Math. Oper. Res 17, 981-1000 (1992)

12. Deza, M.M., Laurent, M.: Facets for the cut cone I. Math. Program. 56, 121-160 (1992)

13. Deza, M.M., Laurent, M.: Geometry of Cuts and Metrics. Springer, Berlin (1997)

14. Diananda, P.H.: On nonnegative forms in real variables some or all of which are nonnegative. Proc. Cambridge Philos. Soc. 58, 17-25 (1962)

15. Dukanovic, I., Rendl, F.: Semidefinite programming relaxations for graph coloring and maximal clique problems. Math. Program. 109, 345-365 (2007)

16. Garey, M.R., Johnson, D.S.: Computers and Intractability: A Guide to the Theory of NP-Completeness. Freeman, New York (1979)

17. Goemans, M.X.: Semidefinite programming in combinatorial optimization. Math. Program. 79, 143-161 (1997)

18. Goemans, M.X., Williamson, D.P.: Improved approximation algorithms for maximum cut and satisfiability problems using semidefinite programming. J. ACM 42, 1115-1145 (1995) 
19. Grötschel, M., Wakabayashi, Y.: Facets of the clique partitioning polytope. Math. Program. 47, 367-387 (1990)

20. Grötschel, M., Wakabayashi, Y.: Composition of facets of the clique partitioning polytope. In: Bodendieck, R., Henn, R. (eds.) Topics in Combinatorics and Graph Theory, pp. 271-284. Physica-Verlag, Heidelberg (1990)

21. Hall, M., Newman, M.: Copositive and completely positive quadratic forms. Proc. Cambridge Philos. Soc. 59, 329-339 (1963)

22. Hill, R.D., Waters, S.R.: On the cone of positive semidefinite matrices. Lin. Alg. Appl. 90, 81-88 (1987)

23. Laurent, M., Poljak, S.: On a positive semidefinite relaxation of the cut polytope. Lin. Alg. Appl. 223(224), 439-461 (1995)

24. Laurent, M., Rendl, F. : Semidefinite programming and integer programming. In: Aardal, K., Nemhauser, G., Weismantel, R. (eds.) Handbook on Discrete Optimization, pp. 393-514. Elsevier, Amsterdam (2005)

25. Letchford, A.N., Sørensen, M.M. : Binary positive semidefinite matrices and associated integer polytopes. In: Lodi, A., Panconesi, A., Rinaldi, G. (eds.) Integer Programming and Combinatorial Optimization XIII. Lecture Notes in Computer Science, vol. 5035, Springer, Berlin (2008)

26. Lovász, L., Schrijver, A.J.: Cones of matrices and set-functions and 0-1 optimization. SIAM J. Opt. 1, 166-190 (1991)

27. Oosten, M., Rutten, J.H.G.C., Spieksma, F.C.R.: The clique partitioning problem: facets and patching facets. Networks 38, 209-226 (2001)

28. Padberg, M.W.: The boolean quadric polytope: some characteristics, facets and relatives. Math. Program. 45, 139-172 (1989)

29. Parrilo, P.A. : Structured semidefinite programs and semialgebraic geometry methods in robustness and optimization. PhD thesis, California Institute of Technology, Pasadena, CA (2000)

30. Sørensen, M.M.: A polyhedral approach to graph partitioning. PhD thesis, Aarhus School of Business (available at www.hha.dk/ $\mathrm{mim} /$ ) (1995)

31. Sørensen, M.M.: A note on clique-web facets for multicut polytopes. Math. Oper. Res. 27, 740-742 (2002) 